\title{
Die Interpunktion im Gesangbuch Jesličky Staré nové písničky von Fridrich Bridelius ${ }^{1}$
}

0. Wie bereits im Titel angedeutet, beschäftigt sich die vorliegende Studie mit der Analyse der Interpunktion in dem alten Druck GESLICZKY | Staré Nowé| Pjfničky/| W nowě Narozenému | Králi KRyftu | GežjJfy | Bethlemfkému | Za Dar| Nowého Léta připfané. (Jesličky. Staré nové písničky, v nově narozenému Králi Kristu Ježíši Bethlemskému za dar nového léta připsané). Es handelt sich um ein kleines Gesangbuch, das nur einmal gedruckt wurde, und zwar im Jahre 1658 in der Prager Akademischen Druckerei der Jesuiten. Autor (in einigen Teilen eher Editor oder „Übersetzer“) war Fridrich Bridelius, eine der markantesten Persönlichkeiten der tschechischen Literaturgeschichte ${ }^{2}$.

0.1. Laut Knihopis ${ }^{3}$ sind die Jesličky in drei Exemplaren erhalten: dem Klementiner (Nationalbücherei in Prag, Signatur 54 F 113), dem Musealen

${ }^{1}$ Dieser Beitrag entstand im Rahmen der Arbeiten an dem Projekt „Jesličky Staré nové písničky (Fridrich Bridelius, 1658) - Eine interdisziplinär angelegte kritische Edition” (GAČR, 406/10/1454). Die Übersetzung aus dem Tschechischen ins Deutsche besorgte Roland Wagner von der Pädagogischen Fakultät der Masaryk-Universität in Brünn.

${ }^{2}$ Innerhalb seines hinsichtlich Genre und Stil äußerst vielseitigen Schaffens genießt v. a. sein sprachlich anspruchsvolles und raffinierte Metaphern gebrauchendes reflexiv-meditatives Gedicht „Co Bůh člověk?” große Anerkennung.

${ }^{3}$ Der „Knihopis českých a slovenských tisků od doby nejstarší až do konce XVIII. stoletî” ist die grundlegende Bibliografie im Bereich des tschechischen Buchdrucks. Sie umfasst alle Erstdrucke und Wiederauflagen, die bis 1800 in tschechischer Sprache erschienen sind. Zurzeit ist sie in aktualisierter Form unter der Bezeichnung „Knihopis digital database” auch elektronisch auf der Webseite des Arbeitsbereiches Klassische Studien am Institut für Philosophie der Akademie der Wissenschaften der Tschechischen Republik unter http://db.knihopis.org/ zugänglich. 
(Bücherei des Nationalmuseums, Signatur 27 D 5) und dem Olmützer Exemplar (Wissenschaftliche Bücherei in Olmütz, Signatur 32.637), das allerdings unvollständig ist: Es endet auf Seite 95; das Manuskript der Jesličky ist leider nicht erhalten. Im vorliegenden Beitrag arbeite ich vorwiegend mit dem Klementiner Exemplar, die anderen beiden Exemplare ziehe ich nur gelegentlich heran. Obwohl die drei erhaltenen Versionen nicht völlig identisch sind (Unterschiede bestehen in der Seitennummerierung, der Anordnung bestimmter Teile und in den Abbildungen), scheinen in der Setzung der Interpunktionszeichen auf den ersten Blick ${ }^{4}$ keine wesentlichen Unterschiede zu bestehen.

Das Gesangbuch enthält nur Liedtexte; der einzige Prosatext ist die zweiseitige Vorrede an den König von Jerusalem. Bereits der Titel des Gesangbuches deutet an, dass die Lieder teils aus älteren tschechischen Gesangbüchern übernommen (staré pisničky), teils neu in die hymnografische Tradition eingeführt werden (nové písničky). Die neuen Lieder machen sowohl originale als auch aus dem Deutschen übersetzte oder adaptierte Kompositionen aus. Ihr Autor/Übersetzer/Editor ist mit größter Wahrscheinlichkeit F. Bridelius selbst. Manchen Liedern ist der Notentext beigegeben, bei anderen wird in einer Anmerkung (stellenweise sehr allgemein, wie z. B. (alt)bekannte Melodie) auf die beabsichtigte Melodie verwiest, wieder andere enthalten keinerlei Informationen zur vorgesehenen musikalischen Umsetzung. Alle Lieder im Gesangbuch sind in continuo, d. h. fortlaufend auf einer Zeile gedruckt.

Die hier vorgelegte Studie ist im Rahmen der interdisziplinären Arbeiten zur Edition des Gesangbuches entstanden, die das Werk zum ersten Mal in allen drei Dimensionen (der literarischen, der musikalischen und der sprachlichen) zugänglich machen soll ${ }^{5}$. Zur Vorbereitung einer adäquaten Edition eines alten Textes muss zunächst die grafische Seite untersucht werden, und zwar v. a. deswegen, weil sich im Neutschechischen (nach einer ganzen Serie von Reformen in der ersten Hälfte des 19. Jh.) ein neues Rechtschreibsystem entwickelt hat. Dieses System unterscheidet sich grundlegend von demjenigen des Mitteltschechischen, welches sich vereinfacht gesagt als „,brüderlich“ bezeichnen lässt. Es entstand am Ende des 16. Jh. in den Druckereien der Böhmischen Brüder, die in der Zeit des Humanismus die Hauptträger der

4 Eine genauere Untersuchung der Übereinstimmungen und Unterschiede in der Interpunktion ist bereits geplant.

${ }_{5}$ An dieser Aufgabe arbeite ich zusammen mit zwei Kollegen: mit der Literaturhistorikerin Dr. Marie Škarpová und dem Musikwissenschaftler Dr. Tomáš Slavický, mit denen das Projekt gemeinsam beantragt wurde und nun durchgeführt wird. 
schriftlichen Tradition eines kultivierten Tschechisch waren, und die hier entstandenen Werke, besonders die Kralitzer Bibel, wurden für die folgenden Jahrhunderte Vorbild für sprachliche Richtigkeit.

Zur Interpunktion in dem Gesangbuch von Bridelius habe ich mich bereits früher sehr allgemein geäußert, und zwar in einem Artikel, der der orthografischen Seite des Gesangbuches insgesamt gewidmet war (Kosek 2010a). In diesem Artikel habe ich v. a. das System der phonematischen Grapheme (der Lettern mit Lautwert) einer Analyse unterzogen. Daher blieb in dieser Studie nicht viel Raum für eine detaillierte Auseinandersetzung mit der Interpunktion, weshalb ich nun zu dieser Frage zurückkehre, und zwar mit dem Ziel einer umfassenden Analyse. Wie bereits gesagt liegt der Sinn der vorliegenden Arbeit darin, eine feste Grundlage zur Festlegung von Transkriptionsregeln für das Gesangbuch zu schaffen. Diese Vorarbeit ist deswegen besonders wichtig, weil die Interpunktion einen neuralgischen Punkt bei der Edition von tschechischen Denkmälern der mitteltschechischen Periode darstellt ${ }^{6}$.

0.2. In meinem Beitrag beschäftige ich mich sowohl mit dem Gesamtinventar der Interpunktionszeichen als auch mit den Regeln, die ihrer Verwendung in dem Gesangbuch von Bridelius zugrunde liegen. Dabei bemühe ich mich, meine Darstellung in den Kontext der Zeit zu stellen: einerseits um zu zeigen, worin das Gesangbuch mit dem zeitgenössischen Usus übereinstimmt, andererseits auch um Fehler zu orten und diese nicht als authentische Erscheinungen der zeitgenössischen Orthografie zu missdeuten ${ }^{7}$. Die Untersuchung fußt auf dem Gesamtrepertoire aller Liedtexte im Gesangbuch, die statistischen Angaben, die in dieser Studie an verschiedenen Stellen gegeben werden, beziehen sich allerdings nur auf eine repräsentative Stichprobe ${ }^{8}$.

${ }^{6}$ Obwohl die Transkriptionsregeln für Denkmäler der frühneutschechischen Epoche, die von dem Wiener Bohemisten J. Vintr (1998) formuliert wurden, die Empfehlung enthalten, in modernen Editionen die ursprüngliche Interpunktion zu belassen, wird diese Regel von den Editoren durchweg durchbrochen. Üblich ist die Anpassung der zeitgenössischen Interpunktion an die neutschechischen Regeln.

7 Dabei kann ich an meine frühere Studien anknüpfen (Ko sek 1999, 2001, 2010b).

8 Die Stichprobe umfasst ungefähr die Hälfte der Lieder, die in den „Jesličky” enthalten sind. Im Einzelnen handelt es sich um die Lieder: „Vesele spívejme; Poslán jest Archanjel k Marii Panně; Anjel Gabryel; Bohu všemohoucímu; Ach živ sem! sotva živý; Již Slunce z Hvězdy vyšlo; Den Božího Narození; Zvěstujem vám radost; Prorokovali Proroci; Nastal nám den veselý; Novou radost zvěstuji vám; Dnes se nám Kristus Pán; Vesel se Nebe; Sem sem dětátko; Krásná Panna; Když Panna plačícího; Radújte se Křestané; Veselé Vánoční hody; Poslyš ó Křestané; Zavítej k nám, Dítě milé; Vítej Slavíčku; Podte sem děti; Hled’ Boha jednoho znáti und Kriste pro naše spasení". 
1. Das Repertoire der Interpunktionszeichen, das in dem hier untersuchten Gesangbuch verwendet wird, stimmt in den Grundzügen mit dem zeitgenössischen Repertoire überein: Verwendet werden Komma „, “, Virgel „/ “, Strichpunkt „; “, Doppelpunkt „: “, Ausrufezeichen „! “, Fragezeichen „? “, Punkt „. " und Klammer „ ( ) “9. Wie man sieht, sind die zeitgenössischen Zeichen in ihrer visuellen Gestalt im Prinzip identisch mit den neuzeitlichen (oder neutschechischen) Interpunktionszeichen. Trotz dieser visuellen Ähnlichkeit sind diese Interpunktionszeichen des Barock mit ihren neutschechischen Fortsetzungen (oder Zwillingen) funktional nicht identisch. Der Unterschied besteht v. a. darin, dass sich die tschechische Interpunktion (in Übereinstimmung mit der europäischen Tradition) bis ins 19. Jh. nach dem Pausenprinzip (oder auch dem rhythmisch-euphonischen Prinzip) ${ }^{10}$ richtete. Dies bedeutet nicht, dass die Interpunktionszeichen lediglich zur Markierung von Sprechpausen für den mündlichen Vortrag von schriftlich fixierten Texten gedient hätten; allerdings entsprach die Interpunktion in höherem Maße der potentiellen mündlichen Realisierung des Satzes, als dies später der Fall war. Die tschechische Interpunktion der frühen Neuzeit (16.-18. Jh.) respektierte im Grunde die dreifache hierarchische Gliederung des Satzes nach semanto-syntaktischen Kriterien (Šlosar 1964, 1966), wie sie sich im Umfeld der mittelalterlichen Rhetorik entwickelt hatte ${ }^{11}$ :

1. Die Einheit höchster Ordnung ${ }^{12}$ ist die period $a$, eine intern strukturierte und semantisch abgeschlossene Einheit, die einem vollständigen Gedanken entspricht (mit perioda wird gewöhnlich ein Satzgefüge bezeichnet);

9 Näheres dazu im Anhang. Objektsprachliches Material wird in dieser Studie, sofern es sich nur um einzelne Zeichen handelt, wegen der größeren Deutlichkeit in Anführungszeichen gesetzt; ansonsten gilt die übliche Praxis, Beispiele durch Kursivdruck zu kennzeichnen.

${ }_{10}$ Diese Situation änderte sich im Laufe der ersten Hälfte des 19. Jh., als sich in der neutschechischen Interpunktion das syntaktische Prinzip durchzusetzen begann.

11 Die vereinfachende Darstellung oben geht von der modernen tschechischen Ausgabe der „Etymologie” von Isidor von Sevilla (in der Übersetzung von D. Korte, vgl. Literaturverzeichnis) aus. Sehr ausführlich geht auf die genannte Problematik die Arbeit Pause and Effect. An Introduction to the History of Punctuation in the West von M. B. Parkes (1992) ein, der sich grundlegende Informationen zur Geschichte der mittelalterlichen Interpunktion (mit Exkursen in die Antike und frühe Neuzeit), besonders zur Tradition in Westeuropa und Großbritannien entnehmen lassen.

12 Bei den Bezeichnungen für die hierarchisch geordneten Strukturabschnitte wird die lateinisch-griechische Schreibweise beibehalten, und dies auch in Fällen, in denen eingedeutschte Varianten (z. B. Periode) zur Verfügung stehen. Der Grund ist, dass sich sonst beim comma eine nicht wünschenswerte Homografie zwischen der Benennung des Zeichens „, , " und der 
2. Die Einheit mittlerer Ordnung ist das colon oder auch das membrum), ein syntaktisch relativ selbständiges Glied einer perioda (oder eher mehrere Glieder einer Periode), das im Hinblick auf Bedeutung und Syntax abgeschlossen ist;

3. Die kleinste Einheit ist das comma (oder auch incisum), d.h. ein Strukturabschnitt (oder eher mehrere Abschnitte), der cola bildet und damit - als syntaktisch unselbständiges Element des Satzes auf der Ebene der Satzkonstituente - die kleinste Einheit des Satzes darstellt. Im Neutschechischen sind die Begriffe comma und Redeabschnitt („promluvový úsek“) äquivalent.

Für diese eher semantisch und syntaktisch definierten Einheiten gilt eine wichtige prosodische Eigenschaft: In der gesprochenen Sprache werden sie durch Pausen hervorgehoben: durch schließende Pausen die perioda und das colon, durch nichtschließende das colon und das comma. Mit diesen Pausen sind die meisten der oben angeführten Interpunktionszeichen assoziiert, v. a. der Beistrich ${ }^{13}$ (Komma sowie Virgel), der Strichpunkt, der Doppelpunkt und der Punkt ${ }^{14}$. Die Interpunktion dient daher nicht primär zur Bezeichnung von zwei verschiedenen Pausentypen, sondern zur Bezeichnung von syntaktischen Grenzen, die durch schließende oder nichtschließende Pausen signalisiert werden. Für den Usus beim Druck von Liedtexten ist dabei spezifisch, dass es zu Modifikationen der zeitgenössischen Interpunktionsregeln kommt, soweit es die Strukturierung der Lieder in Vers und Strophe verlangt (vgl. Kosek 1999, 2002, 2010b).

2. Die Angleichung der Interpunktion an die Versform bzw. an die musikalische Seite des Verses ist besonders bei der Setzung des Beistriches

Benennung des entsprechenden Strukturabschnittes ergeben würde. Nach dem hier eingeführten Usus bezeichnet comma stets den Strukturabschnitt, Komma dagegen das Zeichen.

13 Anm. Übersetzer: In der deutschen Übersetzung ergibt sich hier ein weiteres Problem, dass mit der Synonymie bestimmter Bezeichnungen zu tun hat. Im Tschechischen steht mit dem volkssprachlichen Begriff čárka ein Hyperonym für die beiden Begriffe Virgel (für „/ “) und Komma (für „, ") zur Verfügung, das im Deutschen durch die Synonymie der Begriffe Komma (bundesrepublikanischer Usus) und Beistrich (österreichischer Usus; in der Bundesrepublik veraltet für Komma) wegfällt. Für die Übersetzung dieses Textes führe ich daher (nach Absprache mit dem Autor) folgende Konvention ein: Beistrich bezeichnet als Oberbegriff sowohl „/ “als auch „, , ", Komma hingegen nur „, , “.

${ }^{14}$ Die übrigen Zeichen liefern darüber hinaus Informationen, die aus kommunikativer Sicht relevant sind: Entweder bezeichnen sie die illokutive Kraft des Satzes (Fragezeichen und Ausrufezeichen) oder seine informative Profilierung (Klammern). Nichtsdestotrotz sind auch diese Zeichen mit Pausen verbunden. 
auffällig. Dies ist auch nicht weiter überraschend, weil gerade die Setzung des Beistriches am meisten mit der lautlichen Realisierung des Satzes korrespondiert.

2.1. Im Einklang mit dem Usus der Zeit erscheint der Beistrich in zwei Varianten: dem Komma und der Virgel. Ein Unterschied bei der Verwendung dieser beiden Grapheme besteht im zeitgenössischen Usus dabei gewöhnlich nicht ${ }^{15}$, allerdings setzt sich in den gedruckten Editionen von Liedtexten im Laufe des 17. Jh. allmählich die Tendenz durch, die beiden Varianten des Beistriches funktional zu unterscheiden (Kosek 1999, 2002, 2010b) ${ }^{16}$. Gemäß diesem Trend wird im Druck der Jesličky das $\operatorname{Komma}^{17}$ („, ") in der Versmitte gesetzt, die Virgel („, / “) dagegen am Versende:

Ržka $k$ kež Dwa nebo Třil
zberau Je we Gmenu mém/
gát gJem mezy nimi/
přebýwage s nimi/
po wJeckny Dni_ y nynj/
Swěta do Jkonánj.
$1^{18}$

Lájku nejmjrnau/

a Miloft hognau/

od Boha zwëstugel

že geft Milofti/

plná wyprawuge.

8

Die Unterschiede in der Positionierung der beiden Beistriche zeigt die statistische Auswertung der Belegstellen, die anhand einer Stichprobe aus ungefähr der Hälfte der Liedtexte durchgeführt wurde: Hier erscheint die Virgel in insgesamt 1571 Fällen, wovon 1539 auf Setzungen am Versende entfallen. Das Komma erscheint in insgesamt 122 Belegstellen, am Versende ist es jedoch nur sechsmal belegt, wobei es sich in drei Fällen offenbar um Fehler handelt: Das Komma wurde am Ende einer syntaktischen Einheit am Strophenende gesetzt, d. h. anstelle eines Punktes (s. die Belege weiter unten).

Die Virgel erscheint in der Versmitte dagegen häufiger (statistisch gesehen jedoch weniger häufig als das Komma am Versende: Eine Virgel in der Vers-

${ }^{15}$ J. Konstanc hält in seiner Grammatik Lima linguae Bohemicae allerdings für möglich, dass ein feiner Unterschied in der Distribution der Virgel bestand: Er hält es für ein Zeichen, das eine Mittelstellung zwischen Komma (pưl kolečko ne čárčička taková) und Strichpunkt (media nota) einnahm.

${ }^{16}$ In den Gesangbüchern der tschechischen Exilanten machte sich dieser Unterschied gelegentlich in der typografischen Ausführung des Kommas bemerkbar: Im Versinneren wurde das Komma in abgerundeter Form verwendet, während an das Versende das gerade ausgeführte, schräge Komma trat (Kosek 2002).

17 In den Jesličky nur in Form eines abgerundeten Kommas.

18 Da sich der Aufsatz mit der Analyse der Orthografie befasst, sind die Beispielen präsentiert in einer transliterierten Form. 
mitte ist - umgerechnet auf das Gesamtvorkommen - nur in 2,1 \% der Fälle belegt, während ein Komma am Versende in 4,9 \% der Belegstellen vorkommt). Die Positionierung der Virgel in der Mitte eines Verses ist offenbar durch verschiedene Faktoren motiviert:

a) möglicherweise durch einen Mangel an Lettern für das Komma (auch im Falle von anderen Lettern lassen sich in der damaligen Druckpraxis provisorische Lösungen beobachten, s. Kosek 2010a,

b) durch eine Fehlinterpretation des Strophenschemas durch den Setzer, wie dies z. B. bei dem Lied Krásná Panna vorgekommen ist, wo eine Virgel sogar einen Satz im Versinneren teilt:

KRáfná Panna_ prekráfného yynal

nám porodiła.

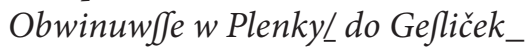
gey połožiła:...

53

c) durch Orientierungsschwierigkeiten des Setzers im Strophenschema: Beim Setzen von Liedern ist unterhalb des Notentextes der Beistrich gelegentlich falsch gesetzt, und dies v. a. dann, wenn der Liedtext auf der folgenden Seite weitergeht. So ist z. B. das Lied O Dobré Noviny auf vier Seiten abgedruckt, von denen drei auch Notentext enthalten. Der letzte Doppelvers befindet sich auf der Vorderseite, und so unterbleibt die Abtrennung durch Beistrich:

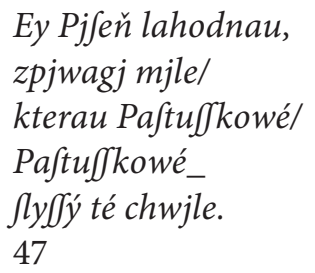

2.2. Der Charakter der Lieder als versifizierte und musikalisch unterlegte Texte bewirkt noch eine weitere Adaption der zeitgenössischen Interpunktionsregeln, die darin besteht, dass das Setzen des Beistriches am Versende obligatorisch wird, sofern der Vers nicht mit einem anderen Interpunktionszeichen schließt:

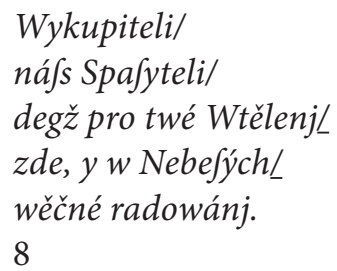

O prediwné NarozenjL pro nafle ypafenj/

Syna Božjho Wtělenj/

gaké ponjženj/

Lidjkému Pokolenj/

pro gich zwelebenj. 


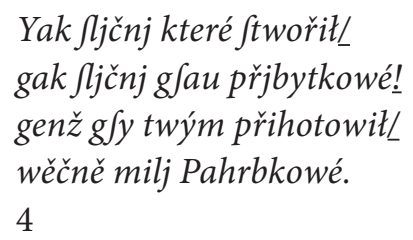
Proč Je Jám rozptytugess?
Proč hledjfs Zbožj marného@
$k d y z ̌$ Boha nemiluge $\int /$

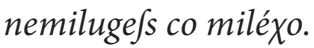
$11-12$

Das obligatorische Setzen eines Beistriches am Versende in einer spezifischen grafischen Form (d. h. in Form einer Virgel) ist deshalb günstig, weil die Lieder, wie bereits erwähnt, in continuo gedruckt sind, d. h. fortlaufend auf einer Zeile. Diese Art des Druckes ermöglicht es, das Papier möglichst effektiv zu füllen und dadurch die Herstellungskosten des jeweiligen Buches für den Verleger zu senken. Die Virgel ermöglicht es dem Leser, sich in einem auf diese Weise gedruckten Text rasch zu orientieren und problemlos die Versgrenze zu identifizieren.

Das konsequente Setzen eines Beistriches am Versende stellt eine Modifikation der zeitgenössischen Pausenregeln dar, die die Setzung des Beistriches ursprünglich nur an der Grenze der kleinsten Einheit im Satz, d. h. nach dem comma vorschreiben (Kosek 1999, 2003): So wie die commata in der freien Rede (unter Berücksichtigung der syntaktischen Ebene des Satzes) die prosodische Grundeinheit des Textes bilden, bildet in der gebundenen Rede (oder eher beim musikalischen Vortrag) der Vers die prosodische (musikalische) Grundeinheit ${ }^{19}$. Im Unterschied zu der prosaischen (oder vielmehr durch kein Metrum gebundenen) Sprache ist der Vers jedoch eine streng normierte Einheit, die im syllabischen System durch eine feste Silbenzahl definiert ist. Diese Versnorm ist äußerst streng, und so kann es dazu kommen, dass selbst die Elemente eines Nominalsyntagmas voneinander getrennt werden, wie das bereits oben angeführte Beispiel von S. 17 der Jesličky zeigt.

19 In der mittelalterlichen lateinischen Poesie bildete sich jedoch eine andere Auffassung heraus. Isidor von Sevilla hält den Vers nämlich für eine Einheit, die das Äquivalent der Periode in der ungebundenen Rede darstellt (Etymologie I, 20,6). Dieser Unterschied in den Auffassungen könnte damit zusammenhängen, dass sich Isidor zur metrischen Poesie äußert, und nicht zu der auf dem syllabischen Prinzip beruhenden Struktur von Liedern. Die mittelalterliche Auffassung vom Vers wird näher in der der Geschichte der Interpunktion gewidmeten Arbeit von Parkes (1992: 98) erläutert: „The grammarians seem to have perceived a verse period as characterized by prosodic continuity: the end of a period coincides with a break in that continuity, and a colon was perceived as a prosodic component of a period. Both period and colon in verse were thus units whose character was determined by different poetic forms and measures: for example, a period may correspond to a single hexameter verse, or to a stanza; a colon may correspond to two feet in a hexameter verse, or to a whole verse in a stanza." 
Dafür, dass das Fehlen des Beistriches am Versende auf Druckfehler zurückgeht, gibt es noch weitere Hinweise:

- Beistriche fehlen auch an Stellen, die nachweislich die Grenze von syntaktischen Einheiten bilden, d. h. an Stellen, an denen der Beistrich unter keinen Umständen fehlen darf:

Rodičko MARYA/
Panenko přečiftá_-
6ud' nám miłoftiwál
po porodu SYNA/
19

Rodičko MARYA/

Panenko přečiftá_

po porodu SYNA/

19
Slzy geho snjmegmel
Plénky, Játky zhřjwegme/
Powiganem Jtahưgme_-
od zléxo ochrańugme/
o milý roztomiłý GežjJ'ku. I:/

50

- Unterschiede bei der Setzung des Beistriches in mehrfach abdruckten, identischen Textabschnitten:

$$
\begin{aligned}
& \text {... mufýss Robátko/ } \\
& \text { do Koljbčičky } \\
& \text { w twrdé Gefličky/ }
\end{aligned}
$$

\author{
...mufýss Robátko/ \\ do Koljbčičky! \\ w twrdé Gefličky.
}

$50-51$

Der Beistrich fehlt am Versende um so häufiger, je größer die Probleme sind, vor die der Setzer durch die Strophenstruktur gestellt wird (dies betrifft v. a. die Lieder Bohu všemohoucímu, Novou radost zvěstuji vám, Krásná Panna und Poslyš ó Křestané): Zum Beispiel zeugt die Interpunktion in dem Lied Poslyšó Křestané von einer Fehlinterpretation des Strophenschemas 6a 6a 6b 6b 4a 6c 6c 4c (sechs-und viersilbige Verse) als Schema mit sieben- und achtsilbigen Versen:

Zě Büh neftwořený/
z łáfky newymluwný/
ráčit Je Wtěliti_
z Panny/ Naroditi/
zde na Zemi/
za nás zapłatiti/
dofti včiniti/
zatracený.

74
To wfle gifti dále/

PaftuJkowéftále/

a řka neme Jkegtel

do Bethléma gdětel

nemellkage_

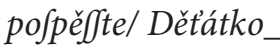

kráfné Pachołátko/

w Jpatném Chléwě.

75

2.3. Mit dem Pausenprinzip hängt die Tendenz zusammen, die Setzung des Beistriches in der Versmitte zu unterdrücken. Diese Tendenz macht sich auch in den Jeslick $y$ bemerkbar, wo syntaktische Einheiten, die nach heute für das Tschechische geltenden Rechtschreibnormen obligatorisch durch Beistrich getrennt werden müssten, nicht durch die Interpunktion kenntlich gemacht sind. Dabei handelt es sich um: 
- Paare von koordinierten Satzgliedern:

Patřmež na Młád’átko/

ležjcý w Gefličkách/

vtéfJené_fjčnél

obwinuté w Plenkách/

gemüžto Nebeftj/

čeft_chwáłu wzdáwagj/

Kưrowé Angeldtj.

19
K Robátku Je Jkłońigmel

Stužbu s łáfkau ftibůgmel

Jawu_TryumfShłáegmel

Srdcem_myfli pleeegmel

ó milý rozromilý GežjđJku. /:/

48

- Wiederholungen:

Wzhuiru_wzhưru a nemelJeg/

do Kofteła бěžl

29

бěž_ochotně бё̌z/

101

- nicht erweiterte Vokative und Interjektionen:

ke mněSe_Synu_přiwiňl

61
ó_tečte_Slzyčky.

77

- nicht erweiterte koordinierte Sätze:

Pána zhřiwagj/

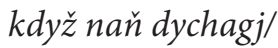

$k d y \check{z}$ řwau_wjtagi/

80
Ey nužwftáwám_nelenugj/

30

- nicht erweiterte Nebensätze (einschließlich von Fällen, in denen das Relativpronomen nicht von einem korrelativ gebrauchten Demonstrativum getrennt wird):

Ey hle Láfky, rozpráwkal
newjm_zdali pochopugj.
1

- Appositionen:

Poděkůgmež Otcy Nebefkému/

y gexo Synu welmi milému/

y Duchu Swatému_Miftru tomu/

3
Stauhau geftit' y Pánem/

ten_genžZemi y Nebem/

nemůž obgat býti/...

24

Marya_čiftá Panenkal

tat' geft geho miłá Matka.

18

Das Pausenprinzip ist in diesen Fällen jedoch für den Leser ungünstig, weil die unterbleibende Kennzeichnung von syntaktischen Einheiten die Orientierung im Text erschwert. Deshalb ist bereits seit Beginn des 17. Jh. eine Tenden z 
zur Syntaktisierung der tschechischen Rechtschreibung zubeobachten (Šlosar 1964, 1966, Králík 1970). Die Konkurrenz beider Prinzipien (des Pausenprinzips und des syntaktischen Prinzips) führt dazu, dass in den oben angeführten Fällen an anderen Stellen im Gesangbuch ein Beistrich gesetzt wird. Besonders koordinierte Satzglieder, Nebensätze und Wiederholungen werden häufig durch Beistrich getrennt. Daraus folgt, dass im orthografischen Usus der Zeit solche Fälle auf zweierlei Weise behandelt werden konnten, was beim heutigen Leser, der an eindeutige Interpunktionsregeln und deren strikte Einhaltung gewöhnt ist, ein Gefühl von Chaos (und beim Editor von existentieller Bangigkeit) hervorruft. Im Folgenden jeweils ein Beispiel für:

- einen durch Beistrich abgetrennten Nebensatz:

Když Je z Panenký narodit/

gfa Pán mocnýs co včinitl

auzkofti w ywětě obljbił/

22

- einen durch Beistrich abgetrennten nicht erweiterten Nebensatz:

Hledjmli na tě, gJem w Rági/

79

- eine durch Beistrich abgetrennte Apposition:

Gedna se zdá 6ýt prácel

Stunce, gedno geho djło/

7

- durch Beistrich abgetrennte nicht erweiterte koordinierte Sätze:

Potmě ležjjs nemáss Swjčky/

73

2.4. Im Geiste der zeitgenössischen Pausen regeln wird der Beistrich im Vergleich zum neutschechischen Usus übermäßig häufig gesetzt, und zwar:

- in der Versmitte, wo er den Satz entsprechend der aktuellen Gliederung in thematischen und rhematischen Bereich einteilt. Diese Untergliederung des Satzes ist besonders in Prosatexten gängig, denn sie respektiert die Gliederung von umfangreichen Sätzen durch Sprechpausen, wie sie bei der gesprochenen Realisierung u. a. im Hinblick auf die Informationsstruktur erfolgen würde (Šlosar 1964, 1966). In Liedtexten wird die Gliederung des Satzes mittels Pausen jedoch obligatorisch durch den Vers realisiert, daher ist in diesen Fällen die Setzung des Beistriches zwischen thematischem 
und rhematischem Bereich recht selten (in meiner Stichprobe finden sich insgesamt nur 10 Belegstellen); bei den entsprechenden Fällen könnte es sich auch um Fehler handeln:

Podzymek geft Sebranj/

we Jměs z rozličných Autori̊l

Hroznuw/Jablek_zbjranj/

nefau dolu y naxoru.

9
Lájka $a_{2}$ Muzyku řjdj/

Sama Struiny nataxuge/

10

- vor den Konjunktionen $a$, i, nebo in kopulativer Funktion:

Syn Božj a Syn Cžłowěkal

62
Ty genž wđechnèm Oděw dáwás/

fám gfy nahý a nic nemáls/

Détátko.

73

Třetj chudých vtijkánj/

Wdow, Syrotkưw fužowánj/

a Cžtwrtý Mzdý zadrženj/

Dělnjkưm ${ }_{2}$ neб vgjmánj.

109

- bei Ausdrücken, die die Bestandteile von Aufzählungen kennzeichnen ${ }^{21}$ :

Duch Swatý Jkrz Cýrkew mluwj/

Třetj, Pofty zachowáweg/

Přikázanj tato, Prwnj/

Cžtwrté, zhřjchů Je wyznáweg/

fwěcený ywátek fwětiti/

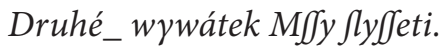

Spráwcy fwému Duchownjmu/

$k$ čafu Welikonočnjmu.

Přigjmeg Welebnau Swátoft/

Páté, бeř Manželfkau Swátoft/

$w$ čafuk tomu nařjzeném/

w Cýrkwi Swáté obyčegném.

107-108

Auch in Fällen, bei denen die Setzung des Beistriches (aus neutschechischer Sicht) überflüssig ist, gilt, dass sich im Text auch häufig Gegenbeispiele, d. h. Belegstellen ohne Beistrich finden. Bei den Konjunktionen $a$ und $i$ lässt sich dabei ein gewisser Zusammenhang zwischen der Länge der durch sie eingeleiteten syntaktischen Einheit und der Setzung des Beistriches feststel-

${ }^{21}$ Wie der eben angeführte Beleg (aus dem Lied Hled'Boha jednoho znáti), der sich auf den Seiten 117-118 findet, zeigt, in dem Fälle mit und ohne Abtrennung durch Komma im Wechsel erscheinen (zur Aufzählung vgl. auch den Abschnitt zum Doppelpunkt). 
len: Ein Beistrich wird bei erweiterten oder längeren Einheiten benutzt, d. h. dann, wenn zwischen den verbundenen Teilen eine Sprechpause zu erwarten ist, während bei nicht erweiterten verbundenen Teilen der Beistrich fehlt:

\begin{tabular}{|c|c|}
\hline $\begin{array}{l}\text { Samá LáJka má Peřil } \\
\text { gjmiž Je wznáfý_a ljtá/ } \\
12\end{array}$ & 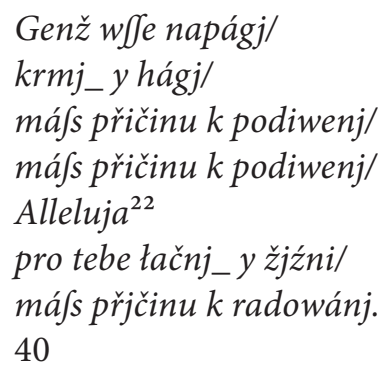 \\
\hline
\end{tabular}

3. Doppelpunkt und Strichpunkt erfüllen die Funktion von Interpunktionszeichen mittlerer Ordnung, d. h. sie machen die interne Organisation der perioda dadurch übersichtlicher, dass sie relativ selbständige Teile, die man im Prosatext cola nennt (vgl. Abschnitt 1), abtrennen. Der Zusammenhang mit der Markierung von cola wird auch in der zeitgenössischen Terminologie deutlich, in der die entsprechenden Zeichen (wie im Deutschen auch heute noch gelegentlich) als colon (Doppelpunkt) und semicolon (Strichpunkt) bezeichnet werden. Bei der Verwendung des Strichpunkts herrschte dabei zunächst eine gewisse Unsicherheit, was dazu führte, dass dieses Zeichen in Drucken des 16. Jh. kaum verwendet wird, und wenn, dann in sehr spezifischer Funktion, z. B. zur Kennzeichnung von Gegensätzen (Králík 1970: 19). Im Laufe des 17. Jh. entwickelte sich zwischen Doppelpunkt und Strichpunkt ein gewisser, wenn auch nicht immer völlig klarer Unterschied: Der Doppelpunkt stellte ein hierarchisch höher stehendes Zeichen als der Strichpunkt dar. D. Šlosar (1999: 36) hat gezeigt, dass dieser Unterschied in den zeitgenössischen Drukken dazu genutzt werden konnte, die lautliche Organisation von komplizierten Satzgefügen anzuzeigen: Der Strichpunkt wurde an die Stelle von Pausen mit nichtschließender Intonation gesetzt, während der Doppelpunkt an Stellen von Pausen mit schließender Intonation gesetzt wurde.

Gewissermaßen als Nachklang der ursprünglichen Unsicherheiten bei der Verwendung des Strichpunktes lässt sich der Umstand interpretieren, dass in den Jesličky so gut wie kein Strichpunkt auftaucht: Im gesamten Text wird dieses Zeichen nur in vier Fällen verwendet. Angesichts der Tatsache, dass zwei der vier aufgefundenen Strichpunkte in der Vorrede, dem einzigen Prosatext in den Jesličky, vorkommen, ist das Fehlen des Strichpunktes wohl eher

${ }^{22}$ Am Versende fehlt hier ein Beistrich. 
stilistischen Faktoren zuzuschreiben. Der syntaktische Bau der Liedtexte, die in den Jesličky enthalten sind, ist nämlich in keiner Weise (besonders was die Satzgefüge betrifft) als kompliziert zu bezeichnen, und daher erübrigt sich die Verwendung von zwei verschiedenen Zeichentypen, die zur Verdeutlichung der internen Gliederung der perioda dienen:

Neygafněgffýmu/ Neyopowrženěgffýmu: Neynepřemoženěgffýmu/ nynj Láfkau přemoženému; Nad Krále Země Neywywýffeněgffýmu/ (a) pro nás w CXléwě Neyfnjženěgffýmu. (b) Neobfáxlému/ (c) Obfaženému: (d) Wywýffenému/ Ponjženému: Welikému Králi nadewffecky Krále/ (e) ted' Maličkému mezy Howady: (f) Welikému Pánu/ (g) Maličkému : (h) Stráfliwému/ (i) Miloftiwému welmi: (k) Newiditedlnému/ (1 1. Tim: 1,) na Zemi fpatřenému: (m) Očekáwánj Národůw/ (n) Poxorffenj Židůw: (o) 1. Cor.

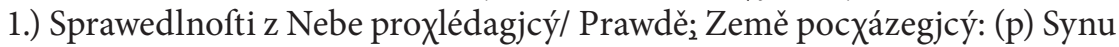

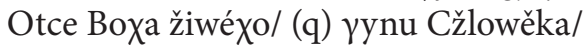

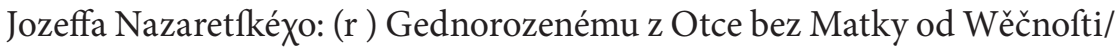
(s) Yynu bez Otce z Matky w plnofti Cžafưw: ( $t$ ) Neywyšffýmu Knězy, podlé Ržádu Melexyfedecxowa/ (u) Králowfkému Kmenu z Pokolenj Judowa: (x) Apoc. 3.) Paftýři/ A (y loan.10.) Beránku: (z) Přjmluwcy/ Orodownjku/...

Da im Text praktisch kein Strichpunkt verwendet wird, erfüllt der Doppelpunkt sowohl die Funktion eines Zeichens, das mit schließenden Pausen assoziiert ist, als auch die Funktion eines Zeichens, das mit nichtschließenden Pausen assoziiert ist. Eine nichtschließende Pause liegt z. B. vermutlich in dem Lied Veselé Vánoční hody vor, in dem der zweite Doppelvers durch einen Doppelpunkt abgeteilt ist, auch wenn dadurch der Prädikator von seinem rechtsstelligen Argument getrennt wird, d. h. zwei syntaktisch eng miteinander verbundene Elemente getrennt werden:

WEfele Wánočnj Hody/

Spjwegte w $\int y$ chni Koledy:

O tom co fe nynjftalo/

že Se Lidem narodiło/

Détátko.
Ont' geft Swěta Spafytelem/

Słowo včiněno Télem:

Syn Božj, a Syn Cžłowěkal

Otec бudaucyho Wěkal

Détátko.

72

In einigen Belegstellen leitet der Doppelpunkt wie im Neutschechischen Aufzählungen (Beleg von S. 109) oder Zitate (Beleg von S. 107) ein:
Cyzý Jau nađfý Hřjchowél
kdy fme my gich piơodowé:
Prwnj geft k Hřjchu raditil
Druhý hřefyti weleti.
109

Cžas Swátku pečůg fwětiti/

Rodiče w luffné cti mjti:

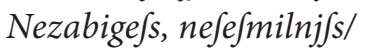

a Krádeže nevčinj/s.

107 
Die Interpretation ergibt sich hier allerdings nur aus den syntaktischen Bezügen der verbundenen Satzteile, der Doppelpunkt fungiert lediglich als Gliederungssignal.

Im Usus der Zeit wurden Doppelpunkt und Strichpunkt auch zur Kennzeichnung der Strophenstruktur eines Liedes verwendet (z. B. in dem Gesangbuch Slaviček rájský von Božan, vgl. Kosek 1999), besonders um die internen Grenzen in dreiteiligen Strophen sichtbar zu machen ${ }^{23}$. In den Jesličky ist die Zeichensetzung in der dreiteiligen Strophe jedoch recht kompliziert (s. unten), und so erscheint der Doppelpunkt hier nur in Ausnahmefällen, wie z. B. in dem nachgedruckten Lied Chtíc aby spal (vermutlich ist der Doppelpunkt hier aus der älteren Druckvorlage übernommen):

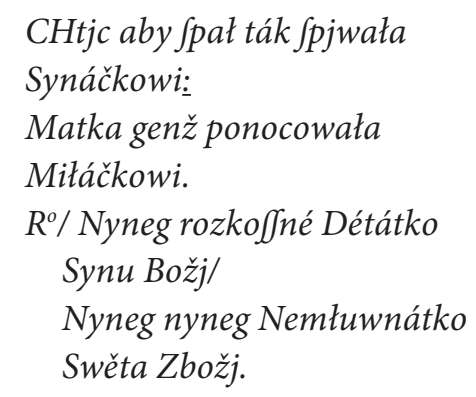

CHtjc aby jpał ták Jpjwała Synákowi:

Matka genžponocowała Miłáčkowi.

Synu Božjl

Swěta Zbožj
Tobě Lůžko gfem vftłała

Spafyteli:

Tworyk twé chwále zwołałal

Stworiteli.

$R^{\circ}$ / Nyneg kráso a Koruno

fwrchowanál

nyneg mitugjcých Ceno

winfowaná.

$71-72$

Ein Doppelpunkt kann in Ausnahmefällen, und zwar bei Liedern, die nicht nach dem dreiteiligen Strophenschema aufgebaut sind, auch zur Signalisierung der regulären Strophenarchitektur herangezogen werden. So ist in dem bereits oben angeführten Lied Veselé Vánoční hody (S. 72) der zweite Doppelvers durchgängig durch einen Doppelpunkt abgetrennt.

4. Zu den Zeichen der obersten Hierarchieebene gehören der Punkt, das Fragezeichen und das Ausrufezeichen. Gewöhnlich begrenzen diese Zeichen genau wie im Neutschechischen Einheiten, die syntaktisch, semantisch und intonatorisch abgeschlossen sind, d. h. Perioden bilden.

4.1. Das Fragezeichen und das Ausrufezeichen, also Zeichen, die die illokutive Kraft des Satzes signalisieren, sind nicht unbedingt mit dem Satzende verbunden und können auch nach den einzelnen Satzgliedern oder

${ }^{23}$ Die dreiteilige Strophe ist die traditionelle Form des tschechischen Liedes. Die Strophe ist dabei in drei Teile eingeteilt: zwei Stollen am Beginn und ein Abgesang am Ende. Die erste und die zweite Stolle (der „Aufgesang“) haben dieselbe Melodie, während sich die Melodie des Abgesangs von derjenigen der beiden Stollen unterscheidet. 
unselbständigen Sätzen innerhalb von Satzgefügen erscheinen. In diesen Positionen haben die Zeichen den Charakter von nicht schließenden Satzzeichen, die den fragenden bzw. exklamatorischen Charakter des jeweiligen Abschnittes der Äußerung anzeigen. Šlosar (1999: 34) wertet sie hier als diakritische Interpunktionszeichen:
Nechcy widěti Nebe/
Nebe geft gednom Zatměnj/
ó Bože! žádám tebel
twému prèekážj widěnj.
14

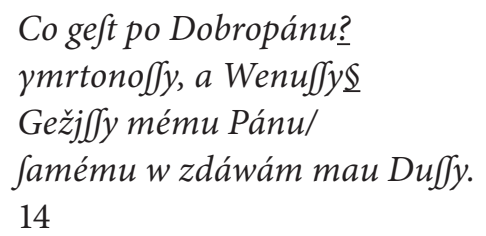

Wie aus dem letzten Beleg ersichtlich ist, hat das Fragezeichen zwei typografische Varianten (s. Anhang). Diese Varianten sind sehr alt und gehen noch auf die mittelalterlichen Anfänge der Interpunktion zurück ${ }^{24}$. Im Text besteht zwischen den beiden Varianten kein wesentlicher Unterschied.

4.2. Im zeitgenössischen Usus gibt es einen engen Zusammenhang zwischen den schließenden Satzzeichen und dem Strophenende, weil die Strophe im Lied das Äquivalent zur perioda in der ungebundenen Rede darstellt (ähnlich wie der Vers das Äquivalent zum comma darstellt). Daher wird im zeitgenössischen Usus das Strophenende obligatorisch durch Punkt, Fragezeichen oder Ausrufezeichen gekennzeichnet, und zwar auch dann, wenn die syntaktische Einheit über das Strophenende hinausgeht. Dies sieht man z. B. in dem Lied Zvěstujeme vám radost, dessen letzte Strophe von der vorausgehenden Strophe durch einen Punkt abgetrennt ist, obwohl sie durch eine Transgressivkonstruktion gebildet wird, die im Tschechischen syntaktisch unselbständig ist:

Rodičko MARYA/
Panenko přečiftá
6ud'nám miłoftiwál
po porodu SYNA/
at'nas w rozkofs Rág/kaul
$v$ wede a dá nám/
radoft fwau Nebefkau.

Rodičko MARYA/

Panenko prěreciftá

oud nám mitoftiwal

po porodu SYNA/

at' nas $w$ rozkofs Rágfkaul

radoft fwau Nebejkau.

\begin{abstract}
Amen Allelujal
Społu Spjwagjce/

Pánu rozenému/

wždy fe kłaněgjce/

s radoftj bez koncel

miłoft v Gežj fel

na wěky magjce.
\end{abstract}

Amè.

19-20

${ }^{24}$ Das Fragezeichen, das ich aus Sparsamkeitsgründen mit römisch I bezeichnet habe (vgl. Anhang), zeichnete sich von Anfang an durch eine gewisse formale Unbeständigkeit aus (Parkes 1992: 40, 306). 
Diese Regel wird in den Jesličky jedoch durchbrochen; z. B. steht in dem Lied Již Slunce z Hvězdy vyšlo am Ende des Verses ein Doppelpunkt, weil am Anfang der folgenden Strophe eine direkte Rede einsetzt (jedoch nicht durch den Doppelpunkt, sondern durch die Transgressivform řka signalisiert; der Doppelpunkt zeigt lediglich an, dass es sich noch um einen Teil derselben syntaktischen Einheit handelt):

Proroctwj Se naplniło/
zdáwna ohłáfJenél
od Prorokůw Duchem Swatým/
nám předpowědénél
pos/lał nám Bůh $z$ Wýfofti
giž Emanuele:
Ržka Panna má poroditil gwěta Spafytele/ a on má wyfwobodit/ Lid od Nepřjtele/ Žiwot z Žiwota wyßet/ бyt’ nám miloft naffet. 17

Auch in anderen Fällen lässt sich in den Jesličky ein Zusammenhang zwischen der Verwendung des Punktes und der Strophenstruktur feststellen. In einigen Liedern teilt der Punkt systematisch die einzelnen Glieder einer dreiteiligen Strophe ab, besonders die zweite Stolle vom Abgesang. Aus dem Vergleich zweier Strophen aus unterschiedlichen Liedern (Nastal nám den veselý \{links\} und Krásná Panna \{rechts\}) ist allerdings ersichtlich, dass auch diese Regel durchbrochen werden konnte:

\begin{tabular}{|c|c|}
\hline Naftał nám den wefelý/ & Protož y my Stwořenj rozumnél \\
\hline z Rodu Králowfkého. & tak хo wjtegme: \\
\hline W němž nám príiflo toho dnel & geho Jlawné Narozenj chwalmel \\
\hline zŽiwota Panenfkého. & takto gey ctěme_ \\
\hline $\begin{array}{l}R^{\circ} / \text { Djtě welmi přediwnél } \\
\text { y také přeradoftnél }\end{array}$ & $\begin{array}{l}R^{\circ} \text { / Otcy Nebejkémul } \\
\text { na nás łajkawému }\end{array}$ \\
\hline w Těle ymrtedłnoftil & za ně děkugjc/ \\
\hline o němž nelze mluwiti/ & a fe radugjc. \\
\hline $\begin{array}{l}\text { myfliti wyprawiti/ } \\
\text { wedlé gexo Bož/twi. }\end{array}$ & 54 \\
\hline
\end{tabular}
23

5. Die direkte Rede wird in den Jesličky durch keinerlei besondere Interpunktionszeichen signalisiert. Sie kann durch Punkt oder Doppelpunkt eingeleitet werden, die Abtrennung kann aber auch ganz unterbleiben:

Dále geft wyznal fám/

řka: Přjklad dal gfem wám/...

${ }^{\star} 1 \mathrm{r}$
Otcowé ywatj wołali/ prołom Nebefa_žádali/ $w$ temnoftech očekáwali/ řkauc. Pane neračiž prodljwati/... 
6. Nur in Ausnahmefällen findet man im Text der Jesličky auch Kla mmern vor. Sie tauchen nur in der Vorrede auf, wo sie verschiedene (teilweise grafische) Funktionen erfüllen: 1. zeigen sie Parenthesen an, d. h. Texteinheiten von geringerer kommunikativer Bedeutung, die Kommentare zum Inhalt des vorher Mitgeteilten oder Ergänzungen in Form von intertextuellen Verweisen darstellen: Newiditedlnému/ (l 1. Tim: 1,2 na Zemi Spatřenému: $\dagger 2 \mathrm{r} ; 2$. signalisieren sie Ergänzungen zum Text: Nevmjm (dále) mluwiti $\uparrow 2 \mathrm{v} ; 3$. markieren sie Buchstaben, die Gliederungspunkte bezeichnen:

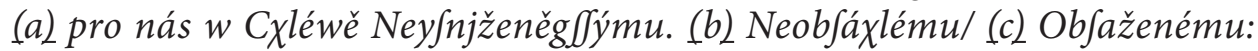
(d) Wywýfenému/... †2r.

7. Neben den Interpunktionszeichen kommen im Text auch grafische Zeichen vor, die die musikalische Umsetzung des Liedes bzw. die interne Strophenstruktur betreffen. Diese Zeichen sind u. a. auch wegen der bereits erwähnten Einrichtung der Liedtexte für den Druck wichtig: Überwiegend sind die Liedtexte nämlich fortlaufend (in continuo) gedruckt.

7.1. Als Zeichen dafür, dass ein Textabschnitt wiederholt werden soll, dient - sofern dieser Textabschnitt nicht erneut abgedruckt ist - gewöhnlich das Symbol „/:/“ oder ,:/:“, in selteneren Fällen auch das Symbol „//“ oder „/./“ (bei den letzten beiden Varianten handelt es sich offensichtlich um Fehler, bei denen ein bzw. zwei Punkte fehlen). Für die Jesličky, die orthografisch sehr variabel sind, ist bezeichnend, dass in einem Lied (O Dobré Noviny, S. 45-47) sogar alle drei Varianten desselben Zeichens nebeneinander erscheinen ${ }^{25}$ :

O Dobré Nowiny/

Pofelftwj Jftiaftné

genž wůbec Andělél

hłáfagj gafnèl

hłafagj gafně

Ey Pjeň lahodnau,

zpjwagj mjlel

kterau Paftufjkowél

Paftufkowé

Sydý té chwjle.

A Stáwa na Nebil

Bohu Intonugj/
A řka nebogte jel milj Paftufjkowél

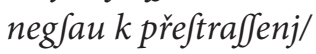

nowiny takowél:/

Y gděte a wěztěl

w Bethlémé maličkým/

nagdete Dětátko/

w Chléwěftudeničkém./:/

An gey čiftá Mátěl

maléxo Koljbal

${ }^{25}$ In diesem Lied wird noch eine weitere Besonderheit der Jesličky deutlich: die Neigung zur Variabilität der Strophenstruktur in einigen Liedern. 


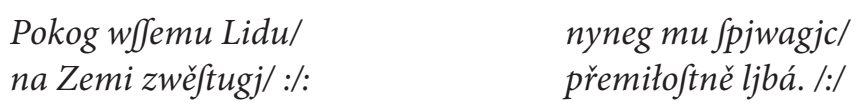

Das betreffende Zeichen dient jedoch auch dazu, die Wiederholung eines Melodieabschnittes anzuzeigen, wie an der letzten Strophe des Liedes Zdrávo bud'ó Nemluvňátko zu sehen ist. Der Vers am Ende der Strophe ist eine Art musikalisches Echo und wird in der letzten Strophe nicht nur einmal, sondern gleich viermal wiederholt, und dies in Form einer Reihung verschiedener Metaphern für das Jesuskind. Eben diese Gradierung am Strophenende wird durch das Zeichen „/:/“ angezeigt (zur Illustration führe ich die erste und die letzte Strophe an):

Zdráwo 6ud’ó Nemtuwñátko/

Gezulátko/

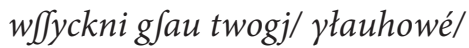

ó Božjké Pańátko.

\author{
Słáwa Nebefkému Otcy/ \\ ó Robátko/

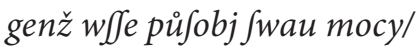 \\ o malé Hołátko. \\ l:/ O miłé Miłátko/ \\ Jjčné Pacholátko \\ rozkoðné Robátko/ \\ drahé Gezulátko.
}

$41-42$

Zu einer weiteren, offenbar fehlerhaften Verwendung des Zeichens, s. auch unten.

7.2. Sehr vage ist die Funktion des Zeichens „R\%“, das in anderen Gesangbüchern aus der zweiten Hälfte des 17. Jh. gewöhnlich zur Kennzeichnung des Abgesangs in einer dreiteiligen Strophe diente. In dieser Funktion kommt „R\%" nur in einigen Fällen vor:

TWá łájka přjlijs weliká

$k$ čemu geft tě prìwedła:

By fe w Gefličky dal kłáftil

miłáčka tě nawedła.

$R^{\circ} /$ Degž at tebel

takgak Jebel

miługi wjce než Jebel

mitugi/ etc.

23-24

\author{
Který nám z drahé pauhé miłoftil \\ toto Spư Sobit/ \\ poflat Syna z weliké miłofti/ \\ aby nás Jpafyz. \\ $R^{\circ} /$ Genž hned od Narozenj/ \\ $w$ žádném potéfenj/ \\ бył náss Pán \\ Kryftus gediný. \\ 53-54
}

Der Setzer scheint sich aber über die Funktion des Zeichens „ $R^{\circ}$ “ nicht im Klaren gewesen zu sein: Offenbar hielt er es für ein allgemeines Wiederholungszeichen, daher erscheint es im Text in ganz unterschiedlichen Funktionen: 
- als Zeichen, das den Refrain kennzeichnet, z. B. in dem Lied Jméno Boží veliké, welches nicht aus einer dreiteiligen Strophe besteht:

GMéno Božj velikél Emanuel/ genžzwěftowat Anget Swatý Gabryet/ dnes Se zgewit w/ermu Lidu Yzrael/ Jkrz Marygi/gemůž Gméno Spafyteł, $R \%$ Naptněno což powědět Gabryeł/ radiogme Sel wejelme Sel

Panna Syna počałal

бez бolefti Kryfta porodiła.
Pán GežjJs narozeny/

бez Muže z čifté Panny/

prawý Bưh w Těle s námi/

Wykupiteł Lidjkého pokolenj/

$R \%$ Naptněno což powědět Gabryeł/

radiogme fe/ wefelme fel

Panna Sýna počałal

бez бolefti Kryfta porodiła

- als Zeichen, das einen Refrain mit Modifikationen im Text kennzeichnet, wie z. B. in dem Lied Co tu stojíte, co se divite, das sich in zwei parallel gebaute syntaktische und semantische Komplexe gliedert, wobei der zweite Komplex leichte Abänderungen im Text aufweist (angeführt sind die erste und dritte Strophe ${ }^{26}$ :

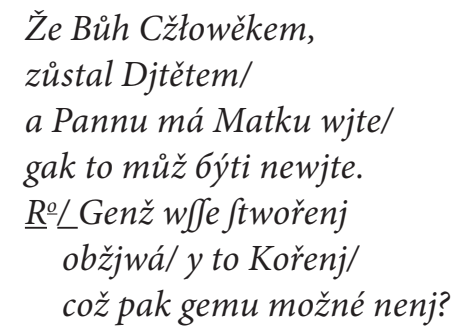

Že Buh Cžłowěkem, züstal Djtètem/

a Pannu má Matku wjtel

gak to můž býti newjte.

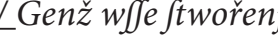

cožpak gemu možné nenj?

\begin{abstract}
Genž Keř zapálit/
wSak gey nespálit/

že Pānu má Mátku wjtel

gak to můž бyti newjte.

Rํㄱ Genž nad Wodami/

Juchýma chodil Noxami/

cožpak gemu možné nenj?

$37-38$
\end{abstract}

- als Zeichen, das darauf aufmerksam macht, dass dieselbe Melodie für beide Stollen einer dreiteiligen Strophe verwendet werden soll ${ }^{27}$. In dieser Funktion kommt das Zeichen allerdings nur in zwei Liedern (Zavitej k nám Dítě milé \{links\} und Slavíčku milý Žáčku \{rechts\}) vor, und auch hier nur in der ersten, abgesetzt unter dem Notentext abgedruckten Stro$\mathrm{phe}^{28}$. Es wäre daher denkbar, dass die Grafik fehlerhaft ist:

${ }^{26}$ Für diese Information bin ich Frau Dr. Marie Škar pová zu Dank verpflichtet.

27 Diese Verwendung steht eigentlich im Widerspruch zur ursprünglichen Funktion des Zeichens „, $\mathrm{R}^{0}$ “", die darin bestand, eine Änderung in der Melodie und nicht deren unveränderte Übernahme anzuzeigen.

${ }^{28}$ Beide Lieder bestehen aus einer dreiteiligen Strophe, ihr Abgesang unterscheidet sich jedoch in keiner Weise. Die im Text oben beschriebene Zeichensetzung erscheint daher als äußerst ungewöhnlich. Auf den Ausnahmecharakter dieser Zeichensetzung deutet auch die grafische Gestaltung des Liedes Slavíčku milý Žáčku hin, dessen erste Strophe 
ZAwjteg k nám Djtě milél

Smutných Kratochwjle:

$R^{\circ} /$ Žes nebylo priffto dýlel

byla Jmutná Chwjle.

Tebe ctj $z$ Nebes wefele/

Andéle Jpjwagj mile/

ó Korůnko mého Cýle/

ó Djtě přemilé.

76
SLawjčku milý Žáčkul

že nebude Zymy/

$R^{\circ}$ / Polefnj Jpjweg Ptáčku/

wjcegj na Zemil

Pofad'Se na Wětwjčku/

giž nebude Zymy/

začni nowau Pjfničku/

že Přjwalu nenj.

80-81

Wie aus der Darstellung oben ersichtlich ist, äußern sich die Unklarheiten hinsichtlich der ursprünglichen Bestimmung von „, $\mathrm{R}^{\circ}$ ” auch darin, dass der Abgesang einer dreiteiligen Strophe oft nicht gekennzeichnet wird, vgl. das Lied Ach kdož podpal společný:

ACh kdož podpat Jpolečný

Syna a Mateřel

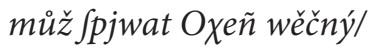

łájka gak welce wrel

Swètu ctná Panenkal

$z$ Žiwota Rodj z Kwětul

malého GežjJka.

64

Unsicherheiten in Bezug auf die Funktion der musikalischen Zeichen weist auch das Lied O Duše má roztomilá auf, in dem der Abgesang (und in der letzten Strophe auch die zweite Stolle) durch das weiter oben beschriebene Wiederholungszeichen „/:/“ bezeichnet sind:

O Duffe má roztomilál

do Bethléma fe бeřmel

$k$ de včiněn čłowěkě

Bůh tám gexo hledegme/:/

Cžłowěk malý Bůh weliký/

ležj naxý Syn Panenky/

Gežjss geft Gméno geho/

Alleluja.
Angełowé profpěwugj/

přewelmi fe radugj/

Paftufkám se vkazugj

Pokog Lidem zwěftugj:_/

w/femu Swětu wygewugj/

že Bůh prìifel prokřikugj/

chwáłu Bohu wzdáwagj/

Alleluja.

45-46

zweimal abgedruckt ist: einmal selbständig unter dem Notentext, ein zweites Mal auf der folgenden Seite ohne Notentext. Das Symbol „R\% “ findet sich allerdings nur im Text unter dem Notentext. 
7.3. Der Anfang jeder Strophe wird im fortlaufenden Druck durch das Strophenzeichen „P/“ angezeigt. Eine Ausnahme bilden abgesetzte Texte unterhalb der Notation, in denen die Strophenzeichen fehlen. Nicht der üblichen Kennzeichnung der Strophe in unserem Gesangbuch entspricht die Zeichensetzung in dem Lied Druhý Slavíček (S. 92-95), in dem die Gliederung in Strophen durch Absätze im Schriftbild angezeigt wird (nach dem Schema ,eine Strophe ist gleich ein Absatz'). Das Strophenzeichen hat im Text prinzipiell zwei Varianten: Es dominiert die Variante in Form eines senkrechten Doppelstriches mit einem Bauch nach links, die sog. Paraphe ${ }^{29}$ (vereinzelt erscheint das Zeichen auch als Schrägstrich mit abgerundetem Ende; hierbei handelt es sich evtl. um schadhafte Typografie: z. B. S. 6, 13 oder 23) ${ }^{30}$. In dem Lied Slavíček Vánoční (S. 81-90) hat das Zeichen die Form eines Pflanzenfiligrans (hedera) ${ }^{31}$. Die doppelte Form des Strophenzeichens lässt (gemeinsam mit anderen grafischen Besonderheiten, vgl. Kosek 2010a) darauf schließen, dass der Druck in verschiedenen Etappen entstanden ist.

Nur in ganz wenigen Fällen erscheint die Paraphe unmotiviert: Gewisse Fälle von Doppelsetzungen einer Paraphe gehen offenbar auf einen Fehler zurück, obwohl im Falle des Liedes Nastal nám den veselý, in dem das Strophenende von Když byl Svět popisován auf Seite 24 durch zwei Paraphen angezeigt wird, auch die Möglichkeit besteht, dass durch diese grafische Gestaltung die interne Gliederung des Liedes verdeutlicht werden soll, da das Lied aus zwei ursprünglich selbständigen Liedern zusammengesetzt ist.

8. Die Interpunktionszeichen können auch noch in anderen Funktionen verwendet werden, die gewöhnlich mit ihren traditionellen, seit dem Mittelalter geläufigen Funktionen als Abkürzungszeichen zusammenhängen:

a) Der Punkt als Abkürzungszeichen

Radi̊gte Se Křeftianél etc ${ }^{32}$.

$67-68$

b) Der Doppelpunkt als Abkürzungszeichen

29 Die Verwendung von Paraphen als Strophensymbole hat eine lange Tradition. Das Zeichen hatte sich bereits im 12. Jh. aus dem initialen C entwickelt, das das lateinische Wort capitulum vertrat und damit - wie die Etymologie des Wortes andeutet - relativ selbständige Texteinheiten kennzeichnete (Parkes 1992: 43).

30 S. Anhang.

31 Vgl. Anhang.

${ }^{32}$ Die Abkürzung etc. hat die damals übliche Gestalt einer Ligatur. 
- in konventionalisierten Fällen: Tim: $1^{\star}$ r, Rozg: 7

- für einen konkreten Textteil, der dem Leser aus dem vorangehenden Kontext bekannt ist, z. B. in dem Lied Novou radost zvěstuji vám, in dem der letzte Doppelvers nur in den zwei ersten Strophen voll ausgesetzt ist; die anderen Strophen sind abgekürzt:

\begin{tabular}{|c|c|}
\hline $\begin{array}{l}\text { Wzhüru wzhüru a neme/fkeg/ } \\
\text { žádnému w tom předku nedeg/ } \\
R^{\circ} \text { / ó Křestiané wftań a neležl } \\
\quad \text { k Dětátku k gefljm, } k \text { Koljbce бěž. }\end{array}$ & 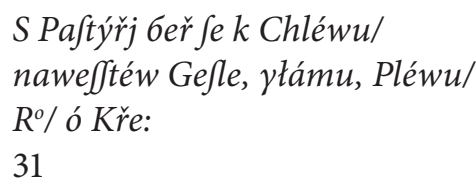 \\
\hline
\end{tabular}

9. Für die Anwendung der zeitgenössischen Rechtschreibregeln ist eine gewisse Großzügigkeit typisch. Neben klaren Fehlern finden sich Fälle, bei denen dieselben syntaktischen Konstruktionen unterschiedlich notiert sind (vgl. oben zur - aus neutschechischer Sicht - überflüssigen bzw. fehlenden Kommasetzung). In dem Lied Bohu všemohoucímu (S. 10) sind z. B. zwei auf Disjunktion beruhende Verse völlig parallel konstruiert und weisen dennoch Unterschiede in der Interpunktion auf; in dem Lied Sem, sem dètátko ist von zwei direkt untereinanderstehenden Vokativen der eine vom zugehörigen Imperativ durch ein Komma getrennt, der andere hingegen nicht (S. 53):

\begin{tabular}{|c|c|}
\hline $\begin{array}{l}\text { Byt'by někdy na Swětěl } \\
\text { nětco Se nedoftáwało/ } \\
\text { бud'w Zymé_nebo w Létěl }\end{array}$ & 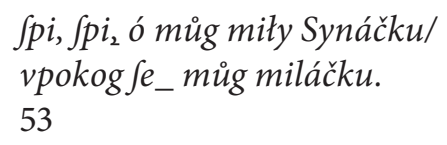 \\
\hline
\end{tabular}

10

Neben dieser inkonsequenten Regelanwendung finden sich auch kla re Fehler:

... Djtě narozeno/

Słowo Tělem včiněno/

Panna porodiła.L

Panenkau zi̊ftała.

$67-68$

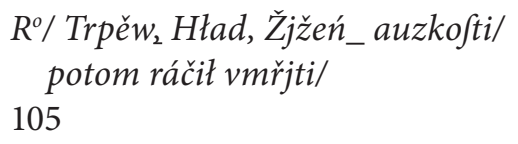

Inkonsequente Regelanwendung zeigt sich auch bei der Kennzeichnung von zu wiederholenden Textabschnitten. So wird in der ersten Strophe des Liedes Prorokovali Proroci das Zeichen für Wiederholung gesetzt, gleichzeitig ist aber die zu wiederholende Passage erneut abgedruckt. Nichtsdestotrotz erscheinen in den weiteren Strophen weder Wiederholungszeichen noch wiederholt gedruckte Textpassagen:

Prorokowali Prorocy/ wzegdet Slunce Sprawedtnofti/
Otcowé ywatj wołali/

prołom Nebefa žádali/ 
By Lid zprođtěn 6ył Temnofti/ ymjrce mezy Bohem a Lidem/ zdáwna žádaný Swatým Dawidem::: zdáwna žádaný Swatým Dawidem. w temnoftech očekáwali/

řkauc. Pane neračiž prodljwatil

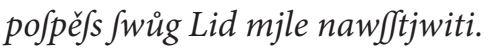

\section{Schluss}

10.1. Die Interpunktion in dem hier untersuchten Gesangbuch von F. Bridelius entspricht in Grundzügen den Interpunktionsregeln der Zeit, die primär auf dem Pausenprinzip und einer dreistufigen Hierarchisierung des Satzes/des Satzgefüges (perioda) beruhen. Da das untersuchte Material aus Liedtexten besteht, richtet sich die Setzung der einzelnen Zeichen zur Textgliederung (mehr oder weniger) auch nach der vorgesehenen musikalischen Umsetzung des jeweiligen Textes (d. h. nach der Vers- und Strophenstruktur der Lieder):

1. Die hierarchisch am tiefsten stehende Einheit der Interpunktion ist der Beistrich, der die kleinste, syntaktisch unselbständige Texteinheit abtrennt: den Vers. Auch wenn der Beistrich bei gebrochenen Versen und übergreifenden Sätzen nur eingeschränkt gesetzt wird, hat die statistische Auswertung des Materials gezeigt, dass auch in diesen Fällen die Setzung des Beistriches deutlich überwiegt.

2. Den cola in der Prosa stehen in Bezug auf die hierarchische Organisation die einzelnen Teile der intern gegliederten Strophen nahe. Um diese voneinander zu trennen, wird stellenweise der Doppelpunkt verwendet (der Strichpunkt kommt im vorliegenden Druck praktisch nicht vor). Da sich der Setzer aber mit der strophischen Struktur der Lieder schwertut, kommt es zu Schwankungen im Gebrauch des Doppelpunktes in dieser Funktion; letztendlich entspricht die Funktion des Doppelpunktes im behandelten Text eher derjenigen, die wir aus Prosatexten kennen: Er zeigt syntaktisch und semantisch autonome Teile einer Periode an.

3. Mit dem Ende der Satzeinheit, d. h. der Periode (perioda), sind die schließenden Zeichen „.“, „!“ und „?“ assoziiert. Im Unterschied zu anderen Gesangbüchern der Zeit ist die Verbindung zwischen Punkt und Strophenende weniger zwingend, so dass auch die internen Grenzen innerhalb einer Strophe (z. B. bei dreiteiligen Strophen) durch Punkte angezeigt werden können. Ausrufezeichen und Fragezeichen können - wie auch sonst im Usus der Zeit - im Versinneren gesetzt werden.

In der Distribution der Beistriche zeigt sich eine weitere Anpassung der Interpunktion an die musikalische Umsetzung der Lieder, die auch aus 
anderen Drucken von Gesangbüchern dieser Zeit bekannt ist: Je nach Position im Vers werden zwei Versionen des Beistriches unterschieden. Dabei steht die Virgel obligatorisch am Versende, während das Komma an anderen Stellen gesetzt wird. Diese Feststellung relativiert ältere Annahmen, denen zufolge es hinsichtlich der Funktion beider Varianten des Beistriches keinen Unterschied gäbe (Šlosar 1964, Králík 1970). Übrigens wird bereits im Anhang zur Grammatik von J. Konstanc (1667, ohne Seitenzahlen) die Möglichkeit angedeutet, die Funktion von Komma und Virgel könne sich unterscheiden: „V starých českých impressích mají dlouhé čárky, které někdy kladou místo dotčeného půl kolečka, někdy pak místo mediae notae a někdy místo dvouch punktů: ovšem zle; nebot jsou cos prostředního mezi půl kolečkem a mediae notae: Semeno po wýchodu Slunce vwadlo / a proto že nemělo kořene vfchlo“33.

Die Setzung des Beistriches im Versinneren wird systematisch unterdrückt: Insbesondere Vokative, Appositionen, kurze Haupt- und Nebensätze oder kurze koordinierte Sätze werden nicht durch Beistrich abgeteilt. Das Fehlen des Beistriches geht in diesen Positionen zweifellos auf das Bemühen zurück, den Vers nicht durch Interpunktionszeichen, die eine Sprechpause anzeigen würden, zu stören und durch Vermeidung des Zeichens die metrische Struktur zu wahren. Bedingt durch den Umstand, dass die Interpunktionsregeln nur sehr allgemein definiert waren und sich neben dem Pausenprinzip allmählich die Tendenz zu einer an der Syntax orientierten Rechtschreibung bemerkbar machte, erscheinen im Text der Jesličky immer auch Gegenbeispiele. Damit soll gesagt sein, dass die Setzung des Beistriches im Versinneren tolerant gehandhabt wurde und Schwankungen im Usus festzustellen sind. Aus dem genannten Grund (Vermeidung der Versteilung durch Interpunktionszeichen) kommen jedoch in unserem Gesangbuch weniger Fälle von (aus neutschechischer Sicht) überflüssiger Setzung des Beistriches vor, als dies in Prosatexten der Zeit der Fall ist. Eine Folge dieses Usus ist jedoch, dass im Versinneren gewöhnlich auch erweiterte Satzglieder nicht durch Beistrich abgetrennt werden und die Setzung auch zwischen Thema und Rhema des Satzes unterbleibt.

33 „In alten tschechischen Drucken kommen lange Striche vor, die manchmal anstelle des erwähnten halben Ringleins, manchmal auch anstelle von mediae notae und manchmal anstelle eines Doppelpunkts gesetzt werden: jedoch fälschlicher Weise; denn sie sind ein Mittelding zwischen halbem Ringlein und mediae notae" (Übers. Roland Wagner). Mit mediae notae sind Strichpunkte gemeint. 


\section{Anhang}

Inventar der Interpunktionzeichen

\begin{tabular}{|c|c|c|}
\hline Termin & $\begin{array}{c}\text { Translitera- } \\
\text { tionzeichen }\end{array}$ & $\begin{array}{c}\text { Photokopien } \\
\text { von der Zeichen }\end{array}$ \\
\hline Komma &, & \\
\hline Virgel & / & \\
\hline Strichpunkt & $;$ & \\
\hline Doppelpunkt & $:$ & \\
\hline Fragezeichen I & \{ & \\
\hline Fragezeichen II & $?$ & \\
\hline Klammern & $($ ) & \\
\hline Pusrufezeichen & & \\
\hline
\end{tabular}


Andere Zeichen nicht-phonematischen Natur

\begin{tabular}{|c|c|}
\hline Strophenzeichen & \\
\hline $\begin{array}{c}\text { Zeichen der Nach- } \\
\text { gesangs } \\
\text { Zeichen der Wie- } \\
\text { derholung }\end{array}$ & \\
\hline
\end{tabular}




\section{Literatur}

Čejka M., Šlosar D., Nechutová J. (Eds.), 1991, Gramatika česká J. Blahoslava, Brno. Isidor Von Sevilla, Etymologie I-III, Übersetzung D. Korte, Praha 2000.

Konstanc J., 1667, Lima linguae Bohemicae. To jest: Brus jazyka českého, Praha.

Ko sek P., 1999, Interpunkce písní kancionálu „Slaviček rájský” J. J. Božana, in: Východočeská duchovní a slovesná kultura v 18. století, Hg. V. Petrbok, R. Lunga, J. Tydlitát, Rychnov nad Kněžnou, S. 9-22.

Ko sek P., 2002, Interpunkce „Hory Olivetské” Matěje Tannera, „Listy filologické“ 125, S. 120-138.

Ko sek P., 2010a, Grafická stránka kancionálu Fridricha Bridelia Jesličky Staré nové písničky, in: Omnibus fiebat omnia. Kontexty života a díla F. Bridelia SJ (1619-1680). Antiqua Cuthna 4, Hg. M. Ška r pová, P. Kosek, T. Slavický, P. Bělohlávková, S. 95-112.

Kosek P., 2010b, Interpunkce českých tištěných kancionálů 17. a 18. století a její transkripce, in: Déjiny českého pravopisu (do r. 1902), Hg. M. Čornejová, L. Rychnovská, J. Zemanová, Brno, S. 250-284.

Králík S., 1970, Větná interpunkce v českých spisech J. A. Komenského, „Slavia“ 39, S. 531-538.

Parkes M. B., 1992, Pause and Effect. An Introduction to the History of Punctuation in the West, Cambridge.

Šlos a D D., 1964, Poznámky k vývoji české interpunkce v 16. století, „Listy filologické“ 87, S. 126-135.

Šlos a r D., 1966, Průřez vývojem staročeské interpunkce, „Listy filologické“ 89, s. 164-169.

Šlosar D., 1977, Poznámky ke dvěma edičním otázkám, „Studia Comeniana et historica - Museum Comenii Hunno Brodense“ 7, S. 65-69.

Šlos a r D., 1999, Středník, in: Tschechisches Barock. Sprache, Literatur, Kultur, Hg. G. Zand, J. Holý, Frankfurt a. M.-Bern-New York-Paris-Wien, S. 33-43.

Voit P., 2006, Encyklopedie knihy, Praha.

Vintr J., 1998, Zásady transkripce českých textů z barokní doby, „Listy filologické“ 111, S. 341-346.

\section{Elektronische Quellen}
„Jesličky, staré nové písničky“ - mezioborově abrufbar unter http://www.bridelius-jeslicky.cz/ koncipovaná kritická edice
Knihopis digital database abrufbar unter http://db.knihopis.org/

